\title{
Germination of Aspergillus fumigatus inside avian respiratory macrophages is associated with cytotoxicity
}

\author{
Lieven Van Waeyenberghe ${ }^{1 *}$, Frank Pasmans ${ }^{1}$, Katharina D'Herde ${ }^{2}$, Richard Ducatelle ${ }^{1}$, Herman Favoreel ${ }^{3}$, \\ Shao-Ji Li ${ }^{1}$, Freddy Haesebrouck ${ }^{1}$ and An Martel ${ }^{1}$
}

\begin{abstract}
Although aspergillosis is one of the most common diseases in captive birds, the pathogenesis of avian aspergillosis is poorly known. We studied the role of avian respiratory macrophages as a first line of defense against avian aspergillosis. The phagocytic and killing capacities of avian respiratory macrophages were evaluated using pigeon respiratory macrophages that were inoculated with Aspergillus fumigatus conidia. On average, 25\% of macrophage-associated conidia were phagocytosed after one hour. Sixteen percents of these cell-associated conidia were killed after $4 \mathrm{~h}$ and conidial germination was inhibited in more than $95 \%$ of the conidia. A. fumigatus conidia were shown to be cytotoxic to the macrophages. Intracellularly germinating conidia were located free in the cytoplasm of necrotic cells, as shown using transmission electron microscopy. These results suggest that avian respiratory macrophages may prevent early establishment of infection, unless the number of A. fumigatus conidia exceeds the macrophage killing capacity, leading to intracellular germination and colonization of the respiratory tract.
\end{abstract}

\section{Introduction}

Fungal infections caused by Aspergillus species are a major cause of morbidity and mortality among captive birds [1]. The filamentous and saprophytic A. fumigatus is the predominant species found in infected birds [2]. This fungus produces huge numbers of airborne conidia, which are found ubiquitously in the environment [3].

The primary route of infection in birds is inhalation of these airborne conidia, resulting in lower respiratory tract colonization [4]. In mammals, the alveolar macrophage is the first line of defense against inhaled conidia [5-7]. In vitro studies show that alveolar macrophages phagocytose and kill attached conidia [6,8-10]. However, in rabbits and mice, conidia of $A$. fumigatus occasionally seem to be able to germinate within alveolar macrophages [11,12].

In comparison to the mammalian respiratory system, a paucity of free respiratory macrophages exists in the avian respiratory system [13-16]. However, scarcity of

\footnotetext{
* Correspondence: Lieven.vanwaeyenberghe@ugent.be

${ }^{1}$ The Department of Pathology, Bacteriology and Avian diseases, Faculty of Veterinary Medicine, Ghent University, Salisburylaan 133 B-9820, Merelbeke, Belgium

Full list of author information is available at the end of the article
}

free respiratory macrophages does not necessarily mean that the avian pulmonary defenses are compromised. Avian respiratory macrophages are innately more competent in phagocytosing particles than mammalian alveolar macrophages and an efficient translocation of subepithelial macrophages to the epithelial surface has been demonstrated $[17,18]$. Moreover, the epithelium and the interstitial macrophages of the atrial and infundibular area play an important role in the removal of particles from the air $[16,19]$.

Due to predominant colonization of the avian lower respiratory tract, avian respiratory macrophages can fulfill an important defensive role during the initial colonization events. Therefore, the aim of this study was to assess the role of the avian respiratory macrophage in avian aspergillosis.

\section{Material and methods}

\section{A. fumigatus growth conditions}

The A. fumigatus isolate, K24, used in the in vitro studies, was obtained from a racing pigeon, which died from pulmonary aspergillosis [20]. Five-day-old cultures of this isolate on Sabouraud dextrose agar (CM0041, Oxoid Ltd., Basingstoke, England) were washed with $5 \mathrm{~mL}$ of $0.01 \%$ 
Tween 20 in RPMI 1640 to harvest A. fumigatus conidia. The conidia were washed three times in $0.01 \%$ Tween 20 in RPMI and the suspension was adjusted to a concentration of $10^{6} \mathrm{~A}$. fumigatus conidia/mL in RPMI 1640 with $1 \%$ glutamine and $1 \%$ pyruvate by haemocytometer count.

\section{Cells}

Pigeon respiratory macrophages were collected according to a method as previously described [21], and maintained in RPMI 1640 supplemented with 1\% glutamine and 1\% pyruvate. Cell sample purity was determined using non specific esterase (Sigma Diagnostics, St. Louis, MO, USA) and Haemacolor (Merck, Darmstadt, Germany) staining.

\section{Conidiacidal ability of pigeon respiratory macrophages}

To assess the killing capacity of avian respiratory macrophages against $A$. fumigatus conidia, pigeon respiratory macrophages were counted and seeded into a 96-well plate at $10^{5}$ macrophages per well. After $2 \mathrm{~h}$ of incubation at $37^{\circ} \mathrm{C}$ and $5 \% \mathrm{CO}_{2}$, the wells were rinsed to remove non adherent cells. The pigeon respiratory macrophages were exposed to $0.2 \mathrm{~mL}$ of $10^{6}$ conidia/mL in RPMI 1640 (+ $1 \%$ glutamine and $1 \%$ pyruvate). The plates were centrifuged at $125 \times g$ for $10 \mathrm{~min}$ to synchronize conidial exposure to the cells. Subsequently, the cells were allowed to ingest the conidia for $1 \mathrm{~h}$ at $37^{\circ} \mathrm{C}$, $5 \% \mathrm{CO}_{2}$. Medium containing non adherent, non phagocytosed conidia was removed, and wells were rinsed three times using $\mathrm{HBSS}$ with $\mathrm{Ca}^{2+}-\mathrm{Mg}^{2+}$ at $37^{\circ} \mathrm{C}$ and fresh medium was added (time point 0 ). The macrophages were then further incubated for $4 \mathrm{~h}$ before cell associated conidia were harvested (time point 4 ). At time points 0 or 4 , plates were frozen at $-70^{\circ} \mathrm{C}$ and rapidly thawed at $37^{\circ} \mathrm{C}$ to lyse the cells and harvest the conidia. Cellular lysis was confirmed by light microscopy. Serial dilutions were performed in sterile medium and immediately plated on Sabouraud dextrose agar. Plates were incubated at $37^{\circ} \mathrm{C}$, and colonies were counted after $24 \mathrm{~h}$ of incubation. Negative control wells did not contain macrophages but were otherwise treated likewise. The percentage killed conidia was calculated by dividing the total number of conidia recovered at $4 \mathrm{~h}$ by the total number of conidia recovered at $0 \mathrm{~h}$ and multiplied by 100 . All tests were performed in triplicate.

\section{Phagocytic capacity of pigeon respiratory macrophages}

To assess the percentage phagocytosed A. fumigatus conidia, pigeon respiratory macrophages were seeded in 24-well plates and exposed to $1 \mathrm{~mL}$ of $10^{5}$ conidia/mL. The plates were centrifuged at $125 \times g$ for $10 \mathrm{~min}$ to synchronize conidial exposure to the cells. After phagocytosis for $1 \mathrm{~h}$, medium containing non adherent, non phagocytosed conidia was removed and the wells were rinsed three times using $\mathrm{HBSS}$ with $\mathrm{Ca}^{2+}-\mathrm{Mg}^{2+}$ at $37^{\circ} \mathrm{C}$.
To discriminate between ingested and adherent conidia, the cells were further incubated with Calcofluor White M2R (Life Technologies Europe BV, Ghent, Belgium), which stains the fungal cell wall of extracellular, but not that of intracellular conidia. For quantification of phagocytosis, the cells were then washed and fixed with $4 \%(\mathrm{v} / \mathrm{v})$ para-formaldehyde for $10 \mathrm{~min}$ at room temperature. Per well, 100 conidia were analyzed for intra- vs. extracellular location using fluorescence microscopy. All tests were performed in triplicate.

\section{Intracellular germination assessed by a fluorescence assay and transmission electron microscopy}

To quantify intracellular germination, pigeon respiratory macrophages were seeded in 24-well plates and exposed to $1 \mathrm{~mL}$ of $10^{5}$ conidia/mL. The plates were centrifuged at $125 \times g$ for $10 \mathrm{~min}$ to synchronize conidial exposure to the cells. After phagocytosis for $1 \mathrm{~h}$, medium containing non adherent, non phagocytised conidia was removed and the wells were rinsed three times using $\mathrm{HBSS}$ with $\mathrm{Ca}^{2+}-\mathrm{Mg}^{2+}$ at $37^{\circ} \mathrm{C}$. To discriminate between ingested and adherent conidia, the cells were further incubated with Calcofluor White M2R. The cells were further incubated for $6 \mathrm{~h}$ and then fixed with $4 \%$ (v/v) para-formaldehyde for $10 \mathrm{~min}$ at room temperature. Per well, 100 conidia were analyzed for germination activity using fluorescence microscopy. The tests were performed in triplicate. To observe the intracellular trafficking and intracellular germination of A. fumigatus conidia electron microscopically, pigeon respiratory macrophages were seeded in 24-well plates at $5 \times 10^{5} / \mathrm{mL}$ and incubated for $2 \mathrm{~h}$ at $37^{\circ} \mathrm{C}$ and $5 \%$ $\mathrm{CO}_{2}$. After the incubation period, the wells were rinsed to remove non adherent cells and subsequently infected with $5 \times 10^{5} \mathrm{~A}$. fumigatus conidia. The wells were then centrifuged at $125 \times g$ for $10 \mathrm{~min}$ at $37^{\circ} \mathrm{C}$ to synchronize conidial exposure to the cells. At time points $0,1 / 2,1,2$, 4 and $8 \mathrm{~h}$, the cell medium was removed and the infected cells were fixated in $4 \%$ formaldehyde containing $1 \% \mathrm{CaCl} 2(\mathrm{w} / \mathrm{v})$ in $0.121 \mathrm{M} \mathrm{Na}$-cacodylate adjusted to $\mathrm{pH} 7$ for $24 \mathrm{~h}$. Then the cells were washed in $\mathrm{Na}$ cacodylate buffer and preserved in the Na-cacodylate buffer. The samples were then divided into smaller portions $\left(1 \mathrm{~mm}^{2}\right)$ with a razor blade, dehydrated in graded ethanol solutions, and embedded in LX112 resin (Ladd Research, Burlington, Vermont, USA). Semithin sections (2 $\mu \mathrm{m})$ were stained with toluidine blue to select the most appropriate regions for ultrathin sectioning. Ultrathin sections of $90 \mathrm{~nm}$ were cut with a diamond knife on a Reichert Jung Ultracut microtome (Jung, Vienna, Austria), mounted on formvar-coated copper grids, and stained with uranyl acetate and lead citrate. The samples were examined under a Jeol EXII transmission electron microscope (JEOL Ltd, Zaventem, Belgium) at $80 \mathrm{kV}$. 


\section{Cytotoxicity assay}

To determine the cytotoxic effect of $A$. fumigatus on avian respiratory macrophages, pigeon respiratory macrophages were seeded in 24-well plates. From each of 5 pigeons, one well with pigeon respiratory macrophages was exposed to $1 \mathrm{~mL}$ of $10^{5}$ conidia/mL and one well was left untreated. The plates were then centrifuged at $125 \times g$ for $10 \mathrm{~min}$. After $6 \mathrm{~h}$ of incubation, the wells were rinsed and exposed to ice cold ethidium monoazide (EMA) solution $(50 \mu \mathrm{g} / \mathrm{mL})$ for $20 \mathrm{~min}$ in the dark. The wells were then exposed to an incandescent lamp (45W) for $10 \mathrm{~min}$. Next, the cells were rinsed and fixed with $4 \%(\mathrm{v} / \mathrm{v})$ paraformaldehyde for $10 \mathrm{~min}$ at room temperature. After washing, the cells were stained with Hoechst $(10 \mu \mathrm{g} / \mathrm{mL})$ for $15 \mathrm{~min}$ at room temperature. Per well, 100 macrophages were analyzed for viability using fluorescence microscopy.

Time-lapse video microscopy of the interaction of pigeon respiratory macrophages with $A$. fumigatus conidia

To verify intracellular germination and cellular lysis, pigeon respiratory macrophages $\left(10^{5} / \mathrm{mL}\right)$ were seeded into a chamber slide system in sterile RPMI 1640 with glutamine medium supplemented with $10 \%$ foetal bovine serum and $1 \%$ penicillin/streptomycin and incubated overnight. After $2 \mathrm{~h}$, the cells were rinsed three times with $\mathrm{HBSS}$ at $37^{\circ} \mathrm{C}$. Cells were inoculated with $1 \mathrm{~mL}$ of $10^{5}$ conidia/mL in RPMI 1640 (+ $10 \%$ foetal bovine serum and $1 \%$ L-glutamine) and the plates were centrifuged at $125 \times g$ for $10 \mathrm{~min}$. The cells were allowed to ingest the conidia for $1 \mathrm{~h}$. Medium containing non adherent, non phagocytosed conidia was removed, and wells were rinsed three times using $\mathrm{HBSS}$ with $\mathrm{Ca}^{2+}-\mathrm{Mg}^{2+}$. To discriminate between ingested and adherent conidia, the macrophages were further incubated with Calcofluor white M2R with a final concentration of $25 \mu \mathrm{M}$. After $30 \mathrm{~min}$, the cells were washed and resuspended in RPMI 1640 (+ 10\% foetal bovine serum and $1 \%$ L-glutamine). The chamber slide dishes were placed on an Olympus IX81 fluorescence microscope (Olympus, Zoeterwoude, The Netherlands) connected to a Cell*M imaging system (Olympus), and equipped with a Hamamatsu B/W Orca camera (Hamamatsu Photonics, Louvain-La-Neuve, Belgium), a Märzhaüser automated microscope table (Märzhaüzer Wetzlar Gmbh, Wetzlar, Germany), and a CellCubator climate chamber (Olympus). Bright-field images of 12 cells were collected every $15 \mathrm{~min}$ for $15 \mathrm{~h}$.

\section{Statistical analysis}

The differences in percentage of apoptotic and necrotic pigeon respiratory macrophages between controls and infected wells were statistically analyzed using a Twosample T test.

\section{Results}

The respiratory system of healthy pigeons showed notable fluctuations in the amount of free avian respiratory macrophages recovered after flushing. The yield of pigeon respiratory macrophages ranged between $3.2 \times 10^{4}$ to $2.0 \times 10^{7}$ with an average of $4.0 \times 10^{6}+/-7.4 \times 10^{6}$ respiratory macrophages per pigeon.

The pigeon respiratory macrophages exhibited a moderate phagocytosis and limited killing capacity in the first hours post incubation. The fluorescence assay showed that of the conidia associated with the macrophages, on average, $25 \%+/-1$ were phagocytosed after $1 \mathrm{~h}$ in pigeon respiratory macrophages and the conidiacidal assay showed that on average, $16 \%+/-6$ of the macrophage-associated conidia were killed after $4 \mathrm{~h}$.

Pigeon respiratory macrophages exposed to A. fumigatus conidia exhibited significantly more necrotic (positive on EMA staining $(P<0.01)$ and significantly less apoptotic (fragmented nucleus visualized using Hoechst staining) cells $(P<0.05)$ than control cells. In infected wells, on average $22.8 \%+/-4.7$ of the cells were necrotic and $5.4 \%+/-2.5$ were apoptotic whereas $9.2 \%+/-3.7$ were necrotic and $11.2 \%+/-4.9$ were apoptotic in the noninfected wells (Figure 1).

Intracellular germination in the cytoplasm of pigeon respiratory macrophages was observed in a small portion of these cells. Six hours after ingestion, the fluorescence assay demonstrated that intracellular germination of conidia occurred in $2 \%+/-1$ of the pigeon respiratory macrophages (Figure 2). TEM was performed to observe the intracellular trafficking of the conidia in macrophages and to verify that conidia can germinate within

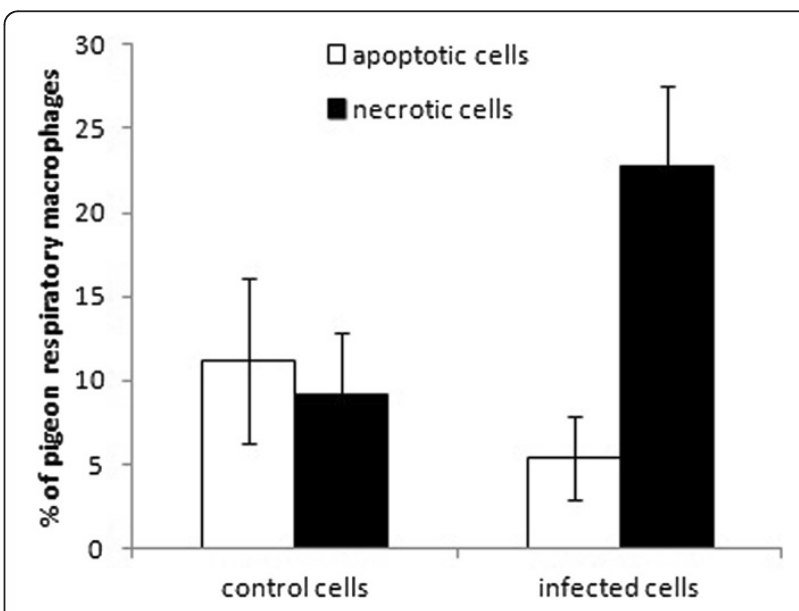

Figure 1 Average percentage +/- standard deviation of apoptotic and necrotic pigeon respiratory macrophages after 6 h of incubation with or without $\boldsymbol{A}$. fumigatus conidia. Cell death was assessed using EMA and Hoechst staining and fluorescence microscopic evaluation of 100 macrophages. The experiment was done 5 times independently. 


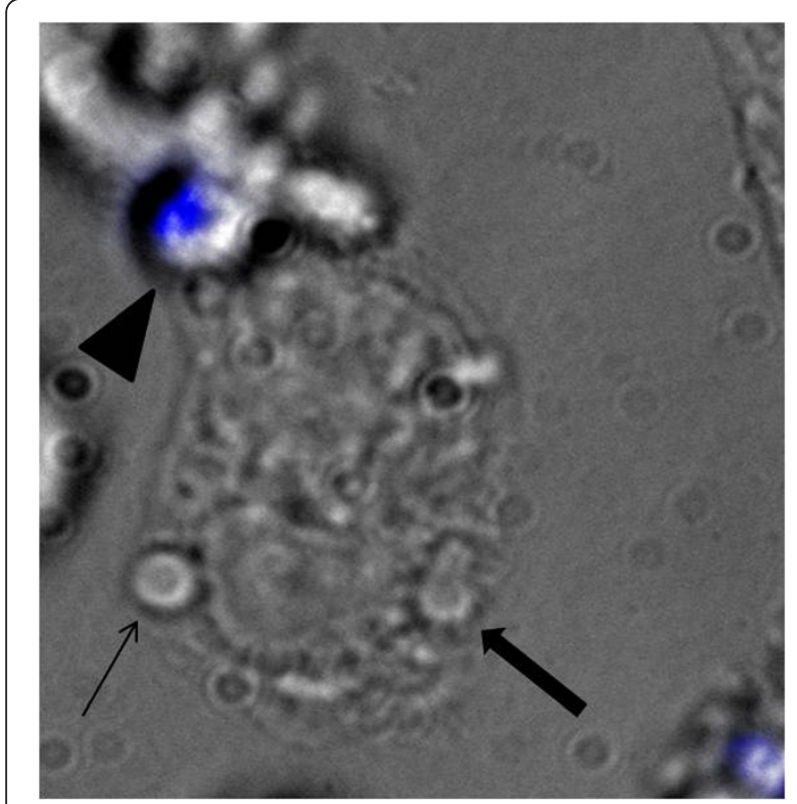

Figure $2 \mathrm{~A}$ pigeon respiratory macrophage with a germinating conidia (thick arrow) and a non-germinating conidia (thin arrow). An overlay is shown of the light microscopic image and the blue fluorescence of non-phagocytised conidia (filled arrowhead) (magnification 600x).

avian respiratory macrophages as observed in the fluorescence assay. After $1 \mathrm{~h}$ of contact between the conidia and the macrophages, some of the conidia were present in phagosomes in the cytoplasm of the macrophages (Figure 3A) while some of the conidia appeared to lay free in the cytoplasm of the cells (Figure 3A). Fusion of the phagosomes, containing conidia, with lysosomes was not observed. Autophagolysosomes and secondary lysosomes were present in multiple macrophages at different time points. Germtube-formation in the cytoplasm of the avian respiratory macrophages was observed $8 \mathrm{~h}$ after the initial contact (Figure $3 \mathrm{~B}+\mathrm{C}$ ). These macrophages were in an advanced stage of degeneration (Figure 3B), leading to oncosis (Figure $3 \mathrm{C}$ ). During time lapse video imaging, intracellular germination was observed and lysis of the cells was frequently observed after ingestion of multiple conidia (data not shown).

\section{Discussion}

This is the first study that reports the phagocytic and killing capacity of avian respiratory macrophages against A. fumigatus conidia. The pigeon respiratory macrophages demonstrated a limited capacity of killing conidia in the first 5 hours post infection, compared to mammals [6,8-10]. However, germination was inhibited in more than $95 \%$ of the cell-associated A. fumigatus conidia, rendering the avian macrophage a highly efficient first line defense against $A$. fumigatus. Inhibition of germination was also observed in alveolar macrophages derived from
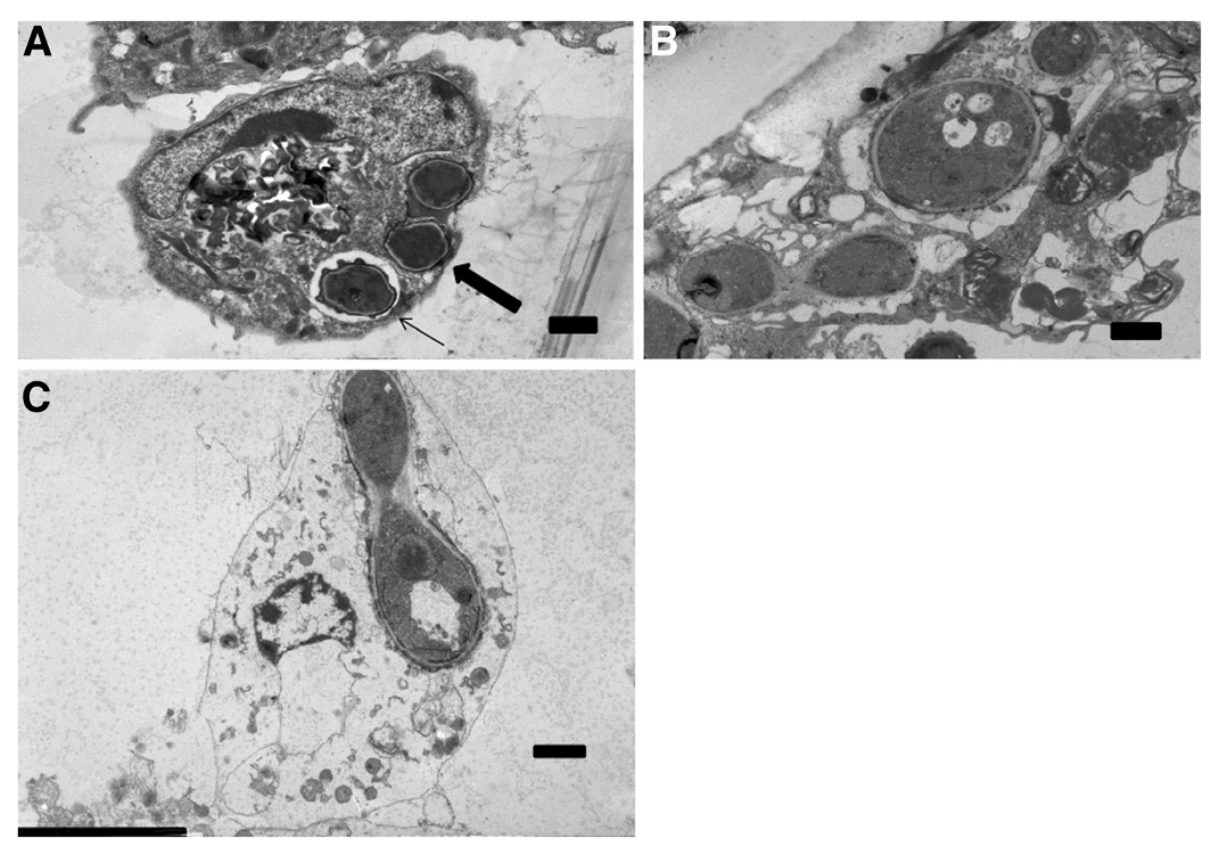

Figure 3 Intracellular germination of conidia inside pigeon respiratory macrophages as revealed by transmission electron microscopy (A to C). Panel A shows internalised conidia inside a vacuole (thin arrow) or inside the cytoplasm (thick arrow) of the macrophage after $1 \mathrm{~h}$. Panels B and C show intracellularly germinating conidia inside a macrophage in an advanced state of degeneration (B) and in oncosis (C) after 8 h. Scalebars in all photos are $1 \mu \mathrm{m}$. 
mice, rabbits and humans [6]. Even though only a limited number of free respiratory macrophages is present in the avian respiratory tract, a rapid influx of activated avian respiratory phagocytes can provide an adequate protection against $A$. fumigatus infections in birds [22].

A small proportion of internalised conidia was still capable of germinating inside the avian macrophages. Intracellular germination was associated with degeneration, oncosis and necrosis of the macrophages. The damage to the macrophages may be elicited by gliotoxin. Gliotoxin is a toxin produced by $A$. fumigatus and is associated with cytotoxicity and cell death in murine macrophages [23]. Taking the limited number of resident respiratory macrophages into account, high environmental exposure to $A$. fumigatus conidia may overwhelm this defense mechanism, which perhaps may lead to colonization of the respiratory tract through intracellular germination of a limited number of conidia that caused macrophage cell death [16,22,24].

In conclusion, this study demonstrates that the majority of A. fumigatus conidia was either killed or their germination inhibited by avian respiratory macrophages. However large amounts of $A$. fumigatus conidia in the respiratory tract can result in intracellular germination and lysis of the phagocytic cells, which may contribute to colonization of the respiratory tract.

\section{Competing interests}

The authors declare that they have no competing interests.

\section{Acknowledgements}

This work was supported by the Institute for the Promotion of Innovation through Science and Technology in Flanders (IWT Vlaanderen), Brussels, Belgium.

\section{Author details}

${ }^{1}$ The Department of Pathology, Bacteriology and Avian diseases, Faculty of Veterinary Medicine, Ghent University, Salisburylaan 133 B-9820, Merelbeke, Belgium. ${ }^{2}$ The Department of Basic Medical Sciences, Faculty of Medicine and Health Sciences, Ghent University, De Pintelaan 185 B-9000, Ghent, Belgium. ${ }^{3}$ The Department of Virology, Parasitology and Immunology, Faculty of Veterinary Medicine, Ghent University, Salisburylaan 133 B-9820, Merelbeke, Belgium.

\section{Authors' contributions}

LWW, AM and FP participated in the design of the study. LWW, KDH, HF and SJL performed the experiments and LWW, RD, KDH, AM and FP analysed the data. LWW, AM, FP, RD and FH wrote the manuscript. All authors read and approved the final manuscript.

Received: 13 January 2012 Accepted: 19 April 2012 Published: 19 April 2012

\section{References}

1. Beernaert LA, Pasmans F, Baert K, Van Waeyenberghe L, Chiers K Haesebrouck F, Martel A: Designing a treatment protocol with voriconazole to eliminate Aspergillus fumigatus from experimentally inoculated pigeons. Vet Microbiol 2009, 139:393-397.

2. Joseph V: Aspergillosis in raptors. Semin Avian Exot Pet Med 2000, 9:66-74.

3. Bain JM, Tavanti A, Davidson AD, Jacobsen MD, Shaw D, Gow NAR, Odds FC: Multilocus sequence typing of the pathogenic fungus Aspergillus fumigates. J Clin Microbiol 2007, 45:1469-1477.

4. Tell LA: Aspergillosis in mammals and birds: impact on veterinary medicine. Med Mycol 2005, 43:71-73.
5. Nessa K, Jarstrand C, Johansson A, Camner P: In vitro interaction of alveolar macrophages and Aspergillus fumigates. Environ Res 1997, 74:54-60.

6. Schaffner A, Douglas H, Braude Al, Davis CE: Killing of Aspergillus spores depends on the anatomical source of the macrophage. Infect Immun 1983, 42:1109-1115.

7. Templeton SP, Buskirk AD, Green BJ, Beezhold DH, Schmechel D: Murine models of airway fungal exposure and allergic sensitization. Med Mycol 2010, 48:217-228.

8. Levitz S, Selsted M, Ganz T, Lehrer R, Diamond R: In vitro killing of spores and hyphae of Aspergillus fumigatus and Rhizopus oryzae by rabbit neutrophil cationic peptides and bronchoalveolar macrophages. J Infect Dis 1986, 154:483-489.

9. Marr KA, Koudadoust M, Black M, Balajee SA: Early events in macrophage killing of Aspergillus fumigatus conidia: new flow cytometric viability assay. Clin Diagn Lab Immunol 2001, 8:1240-1247.

10. Schaffner A: Macrophage-Aspergillus interactions. Immunol Ser 1994, 60:545-552.

11. Behnsen J, Narang P, Hasenberg M, Gunzer F, Bilitewski U, Klippel N, Rohde M, Brock M, Brakhage AA, Gunzer M: Environmental dimensionality controls the interaction of phagocytes with the pathogenic fungi Aspergillus fumigatus and Candida albicans. PLoS Pathog 2007, 3:138-151.

12. Kurup VP: Interaction of Aspergillus fumigatus spores and pulmonary alveolar macrophages of rabbits. Immunobiology 1984, 166:53-61.

13. Ficken MD, Edwards JF, Lay JC: Induction, collection, and partial characterization of induced respiratory macrophages of the turkey. Avian Dis 1986, 30:766-771.

14. Holt PG: Alveolar macrophages. I. A simple technique for the preparation of high numbers of viable alveolar macrophages from small laboratory animals. J Immunol Methods 1979, 27:189-198.

15. Maina JN, Cowley HM: Ultrastructural characterization of the pulmonary cellular defences in the lung of a bird, the rock dove, Columba livia. Proc R Soc Lond B 1998, 265:1567-1572.

16. Nganpiep L, Maina JN: Composite cellular defence stratagem in the avian respiratory system: functional morphology of the free (surface) macrophages and specialized pulmonary epithelia. J Anat 2002, 200:499-516.

17. Kiama SG, Adekunle JS, Maina JN: Comparative in vitro study of interactions between particles and respiratory surface macrophages, erythrocytes, and epithelial cells of the chicken and the rat. J Anat 2008, 213:452-463.

18. Mutua PM, Gicheru MM, Makanya AN, Kiama SG: Comparative quantitative and qualitative attributes of the surface respiratory macrophages in the domestic duck and the rabbit. Int J Morphol 2011, 29:353-362.

19. Reese $\mathrm{S}$, Dalamani G, Kaspers B: The avian lung-associated immune system: a review. Vet Res 2006, 37:311-324.

20. Beernaert LA, Pasmans F, Haesebrouck F, Martel A: Modelling Aspergillus fumigatus infections in racing pigeons (Columba livia domestica). Avian Pathol 2008, 37:545-549.

21. Pasmans F, Baert K, Martel A, Bousquet-Melou A, Lanckriet R, De Boever S, Van Immerseel F, Eeckhaut $\mathrm{V}$, de Backer P, Haesebrouck F: Induction of the carrie state in pigeons infected with Salmonella enterica subspecies enterica serovar Typhimurium PT99 by treatment with florfenicol: a matter of pharmacokinetics. Antimicrob Agents Chemother 2008, 52:954-961.

22. Toth TE: Nonspecific cellular defense of the avian respiratory system: a review. Dev Comp Immunol 2000, 24:121-139.

23. Watanabe A, Kamei K, Sekine T, Higurashi H, Ochiai E, Hashimoto Y, Nishimura K: Cytotoxic substances from Aspergillus fumigatus in oxygenated or poorly oxygenated environment. Mycopathologia 2004, $158: 1-7$

24. Toth TE, Siegel PB: Cellular defense of the avian respiratory tract: paucity of free-residing macrophages in the normal chicken. Avian Dis 1985, 30:67-75.

doi:10.1186/1297-9716-43-32

Cite this article as: Van Waeyenberghe et al: Germination of Aspergillus fumigatus inside avian respiratory macrophages is associated with cytotoxicity. Veterinary Research 2012 43:32 